\title{
Depression and the Risk of Stroke Morbidity and Mortality: A Meta-analysis and Systematic Review
}

\author{
Dr. An Pan, PhD, Dr. Qi Sun, MD, ScD, Dr. Olivia I. Okereke, MD, SM, Dr. Kathryn M. \\ Rexrode, MD, and Dr. Frank B. Hu, MD, PhD \\ Department of Nutrition (Drs Pan, Sun, Hu) and Epidemiology (Drs Okereke, Hu), Harvard School \\ of Public Health, 655 Huntington Avenue, Boston, MA 02115, United States; Channing \\ Laboratory (Drs Sun, Okereke, Hu) and Division of Preventive Medicine (Dr Rexrode), \\ Department of Medicine, and Department of Psychiatry (Dr Okereke), Brigham and Women's \\ Hospital and Harvard Medical School, Boston, MA 02115, United States
}

\begin{abstract}
Context-Several studies have suggested that depression is associated with an increased risk of stroke; however, the results are inconsistent.
\end{abstract}

Objective-To conduct a systematic review and meta-analysis of prospective studies assessing the association between depression and risk of developing stroke in adults.

Data Sources-A search of MEDLINE, EMBASE, and PsychINFO databases (to May 2011) was supplemented by manual searches of bibliographies of key retrieved articles and relevant reviews.

\begin{abstract}
Study Selection-We included prospective cohort studies that reported risk estimates of stroke morbidity or mortality by baseline or updated depression status assessed by self-reported scales or clinician diagnosis.
\end{abstract}

Data Extraction-Two independent reviewers extracted data on depression status at baseline, risk estimates of stroke, study quality, and methods used to assess depression and stroke. Hazard ratios (HRs) were pooled using fixed-effect or random-effects models when appropriate.

Associations were tested in subgroups representing different participant and study characteristics. Publication bias was evaluated with funnel plots and Begg test.

Results-The search yielded 28 prospective cohort studies ( $\mathrm{n}=317540$ participants) that reported 8478 stroke cases (morbidity and mortality) during a follow-up period ranging from 2 to 29 years. The pooled adjusted HRs were 1.45 (95\% confidence interval $[\mathrm{CI}], 1.29-1.63 ; P$-for-

Correspondence to: Frank B. Hu, MD, PhD, Harvard School of Public Health, 655 Huntington Avenue, Boston, MA 02115, United States; Phone: 617432 0113; Fax: 617432 2435; frank.hu@ channing.harvard.edu.

Disclosures: All authors have completed and submitted the ICMJE Form for Disclosure of Potential Conflicts of Interest and none were reported.

Author Contributions: Drs. Pan and Hu have full access to the data in this study and take complete responsibility for the integrity of the data and the accuracy of the data analysis.

Study concept and design: Pan, Rexrode, $\mathrm{Hu}$.

Acquisition of data: Pan, Sun, Hu.

Analysis and interpretation of data: Pan, Sun, Okereke, Rexrode, Hu.

Drafting of the manuscript: Pan.

Critical revision of the manuscript for important intellectual content: Sun, Okereke, Rexrode, Hu.

Statistical analysis: Pan, Sun.

Obtaining funding and Study supervision: Rexrode, $\mathrm{Hu}$.

Administrative, technical, or material support: Rexrode, $\mathrm{Hu}$.

Final approval: Pan, Sun, Okereke, Rexrode, Hu. 
heterogeneity $<0.001$; random-effects model) for total stroke, $1.55(1.25-1.93 ; P$-for-heterogeneity $=0.31$; fixed-effects model) for fatal stroke ( 8 studies $)$, and $1.25(1.11-1.40 ; P$-for-heterogeneity $=$ 0.34 ; fixed-effects model) for ischemic stroke (6 studies). The estimated absolute risk differences associated with depression were 106 cases for total stroke, 53 cases for ischemic stroke, and 22 cases for fatal stroke per 100000 individuals per year. The increased risk of total stroke associated with depression was consistent across most subgroups.

Conclusion-Depression is associated with a significantly increased risk of stroke morbidity and mortality.

\section{INTRODUCTION}

Stroke is a leading cause of death and permanent disability, with significant economic losses due to functional impairments. ${ }^{1}$ Depression is highly prevalent in the general population, and it is estimated that $5.8 \%$ of men and $9.5 \%$ of women will experience a depressive episode in a 12-month period. ${ }^{2}$ The lifetime incidence of depression has been estimated at over $16 \%$ in the general population. ${ }^{3}$ Depression have been associated with increased risks of diabetes, ${ }^{4}$ hypertension, ${ }^{5}$ and cardiovascular disease. ${ }^{6}$ However, whether depression increases the future risk of stroke remains unclear.

A number of studies have assessed the association between depression and subsequent risks of stroke morbidity and mortality, suggesting that depression could be a modifiable risk factor for stroke. ${ }^{7-8}$ A previous meta-analysis that focused on cardiovascular outcomes pooled results from 10 studies published before 2005 as a secondary analysis and reported a positive association between depression and risk of stroke. ${ }^{9}$ Since then many more studies have been published, which allow more detailed analysis of the association between depression and stroke morbidity and mortality. Therefore, we conducted a systematic review and a meta-analysis of prospective cohort studies to describe the association between depression and future risk of total and subtypes of stroke.

\section{METHODS}

\section{Search Strategy}

We conducted a systematic literature search (up to May 2011) of MEDLINE, EMBASE, and PsychINFO for studies describing the association between depression (defined by selfreported scales or clinician diagnosis) and stroke morbidity and mortality. In addition, we searched the reference lists of all identified relevant publications, and relevant reviews. ${ }^{7-9}$ Only papers published in English language were considered. Two search themes were combined using the Boolean operator "and". The first theme, depression, combined exploded versions of Medical Subject Headings (MeSH) depression, depressive disorder, or depressive disorder, major. The second theme, stroke, combined exploded versions of MeSH terms stroke, cerebrovascular disorders, or intracranial embolism and thrombosis.

\section{Selection Criteria}

Two investigators (A.P. and Q.S.) independently assessed literature eligibility; discrepancies were resolved by consensus. Articles were considered for inclusion in the systematic review if: (1) the authors reported data from an original, peer-reviewed study (i.e., not review articles, or meeting abstracts); (2) the study was a cohort study (prospective cohort or historical cohort) consisting of non-institutionalized adults (>18 years old); and (3) the authors reported the risk estimates of stroke morbidity or mortality in depressed participants compared with non-depressed individuals. We used broad inclusion criteria for studies, including all types of stroke (total, fatal, nonfatal, ischemic, and hemorrhagic) and depression status (assessed by different scales or clinical diagnosis). We identified articles 
eligible for further review by performing an initial screen of identified titles or abstracts, followed by a full-text review.

\section{Data Extraction}

We extracted the following information about the studies: study characteristics (study name, authors, publication year, journal, study site, follow-up years, and number of participants), participants' characteristics (mean age or age range, gender), main exposure depression (self-reported scales or clinician diagnosis, assessed at baseline or updated), main outcome stroke (morbidity or mortality, types, assessed by self-report, death certificates or medical records), and analysis strategy (statistical models, covariates included in the models). Quality assessment was performed with consideration of the following aspects: study design, response rate, follow-up rate, follow-up years, exposure and outcome measurements, statistical analysis, and generalizability to other populations (eTable 1).

\section{Data Synthesis}

The Hazard Ratios (HRs) were used as the common measure of association across studies, and the relative risks (RRs) were considered equivalent to HRs. If the result on total stroke were not available, we used data from ischemic stroke, non-fatal stroke or fatal stroke (in the sequential order) as a surrogate for total stroke. Forest plots were produced to visually assess the HRs and corresponding 95\% confidence intervals (CIs) across studies. Heterogeneity of HRs across studies was evaluated by the Cochrane Q statistic $(P<.10$ was considered indicative of statistically significant heterogeneity) and the $I^{2}$ statistic (values of $25 \%, 50 \%$ and $75 \%$ were considered to represent low, medium and high heterogeneity respectively). ${ }^{10-11}$ The HRs were pooled using the fixed-effect model if no or low heterogeneity was detected, or the DerSimonian and Laird random-effects model otherwise, ${ }^{12}$ and the weights were equal to the inverse variance of each study's effect estimation. The possibility of publication bias was evaluated using the Begg test and visual inspection of a funnel plot. ${ }^{13-14}$ The Duval and Tweedie nonparametric trim-and-fill procedure was used to further assess the possible effect of publication bias in our metaanalysis. ${ }^{15}$ Moreover, stratified analyses and sensitivity analyses were performed to evaluate the influences of selected study and participant characteristics on study results. The analyses were performed with Stata statistical software version 9.2 (StataCorp, College Station, Texas). $P$ values were 2 -sided with a significance level of .05 .

We calculated absolute risk differences associated with depression by multiplying the background incidence rate of stroke in the general US population with (estimated HR-1). Population attributable risk (PAR) was calculated based on the following equation: PAR $\%=100 \times \mathrm{Pe}(\mathrm{HR}-1) /(\mathrm{Pe}[\mathrm{HR}-1]+1)$, where $\mathrm{P}_{\mathrm{e}}$ is the prevalence of the exposure (depression) in the population and HR was derived from this meta-analysis.

\section{RESULTS}

\section{Literature Search}

The search strategy identified 7642 unique citations. After the first round screening based on titles and abstracts with the aforementioned criteria, 301 articles remained for further evaluation. After examining those articles in more detail, 276 articles were excluded for reasons shown in Figure 1. Another 2 studies were retrieved from the reference lists, ${ }^{16-17}$ and one was from our recent publication. ${ }^{18}$ In total, 28 articles were included. ${ }^{16-43}$

Among these 28 articles, 8 studies specifically reported results on fatal stroke, ${ }^{16-17,21,26-27,29,33,38} 3$ studies on non-fatal stroke, ${ }^{26,31,38} 6$ studies on ischemic stroke, ${ }^{16,18,24,26,32,35}$ and 2 studies on hemorrhagic stroke. ${ }^{18,24}$ Six studies ${ }^{18,28,32,37,40,42}$ 
reported the crude association between antidepressant medication use and total stroke risk (Wassertheil-Smoller et al ${ }^{28}$ reported the results in a separate paper ${ }^{44}$ ).

\section{Study Characteristics}

Characteristics of the 28 selected studies are shown in Table 1 . The total number of participants included in this meta-analysis was 317540, with 8478 reported stroke outcomes (one study did not report the number of stroke cases ${ }^{19}$ ). The studies varied with regard to how results were presented. Two studies reported results separately by age group: $<65$ and $\geq 65$ years old (Salaycik et al. ${ }^{34}$ ), 65-74 and $\geq 75$ years old (Avendano et al. ${ }^{30}$ ); two studies reported their results separately by baseline history of cardiovascular disease, ${ }^{28,40}$ with one study providing unpublished data for the total sample $;{ }^{40}$ three studies provided results stratified by gender along with the results from total samples; ${ }^{35,37,41}$ With regard to study location, most of the studies were from US or European countries. Three studies were conducted in Japan, ${ }^{24,27,33}$ one in Australia, ${ }^{16}$ and one in Taiwan, ${ }^{36}$ and one was an international collaboration. ${ }^{43}$ The study samples ranged from 401 to 93676 , and the followup durations ranged from 2 to 29 years. Most of the studies comprised both men and women, while two studies included only men, ${ }^{26,29}$ and three studies only women. ${ }^{17-18,28}$

In most of the studies, depression was measured by self-reported scales, such as Center for Epidemiologic Studies Depression Scale, ${ }^{16,20,25,28-30,32,34-35,40-41}$ Zung's Self-Rating Depression Scale, ${ }^{24,33} 30$-item General Health Questionnaire, ${ }^{26-27}$ Geriatric Depression Scale, ${ }^{17,43}$ Beck Depression Inventory, ${ }^{42}$ Human Population Laboratory Depression Scale, ${ }^{21}$ 9-item Patient Health Questionnaire, ${ }^{39}$ and 5-item Mental Health Index. ${ }^{18}$ Four studies used the Diagnostic Interview Schedule (DIS) to define depression as the exposure, ${ }^{23,36-38} 2$ studies included antidepressant medication use as a component of depression definition, ${ }^{18,34}$ and 4 studies used combined methods. ${ }^{18,33-34,40}$ The depression status was only measured at baseline in the majority of studies, while 3 studies used updated depression assessments. ${ }^{18,21,33}$ In most of the studies, stroke was assessed by death certificates or medical records, and some studies combined self-reported measures with medical records; only one study relied solely on self-reported outcomes. ${ }^{41}$ Three studies included outcomes comprised of stroke and transient ischemic attack. ${ }^{16,34,39}$ Baseline stroke cases were not excluded in 7 studies; ${ }^{16-17,20,27-28,39,43}$ we included those studies in the main analysis, but conducted a stratified analysis by presence or absence of baseline stroke cases.

Adjusted HRs could be determined for most studies, except that two studies reported the crude results without adjustment (eTable 2). ${ }^{20,33}$ Most of the results were adjusted for age (25 studies), smoking status (20 studies), BMI (14 studies), alcohol intake (9 studies), physical activity ( 7 studies), and comorbidities ( 23 studies; such as diabetes, hypertension, and coronary heart disease).

\section{Depression and Risk of Stroke Morbidity \& Mortality}

Among the 31 reports from the 28 studies of results on total stroke, the majority of studies reported a positive association (i.e., HR>1.00), with 14 of them being statistically significant. Only four studies reported $\mathrm{HR}<1.00$ but not statistically significant. A moderate to high heterogeneity was detected with an $I^{2}=66.0 \%$ (Cochrane Q statistic $=88.1, P<.001$ ), the HR from random-effects model was 1.45 (95\% CI, 1.29-1.63) (Figure 2). A sensitivity analysis of omitting one study in each turn showed that Lee et al's study ${ }^{36}$ had the largest influence on the results: the pooled HR without this study was 1.36 (95\% CI, 1.24-1.49). Another sensitivity analysis, in which we excluded studies that imputed the risk estimates from other stroke outcomes (ischemic/non-fatal/fatal stroke) if data on total stroke were not available, revealed similar results (HR, 1.46; 95\% CI, 1.26-1.69; 23 reports from 20 studies; $I^{2}=73.7 \%$; random-effects model). We conducted a secondary analysis to combine the 
unadjusted results on the association between antidepressant medication use and stroke risk, and the HR was 1.41 (95\% CI, $1.25-1.59 ; I^{2}=0 \%$; eFigure 1$)$.

Fatal stroke results were available from 8 studies with a pooled HR of 1.55 (95\% CI, 1.251.93) from fixed-effect model (Figure 3A). A modest heterogeneity was found with an $I^{2}=15.8 \%$ (Cochrane Q statistic $=8.32, P=.31$ ). Most of the studies found a HR above 1.00 except one study with an observed HR of $0.45 .{ }^{38}$ Ischemic stroke results were available from 6 studies with a pooled HR of 1.25 (95\% CI, 1.11-1.40; fixed-effect model). A low heterogeneity was found with an $I^{2}=12.3 \%$ (Cochrane Q statistic=5.70, $P=.34$ ). Similar sensitivity analyses for fatal stroke and ischemic stroke did not appreciably change the results (data not shown). Results for non-fatal stroke and hemorrhagic were not significant (1.21 [95\% CI, 0.91-1.62] and 1.16 [95\% CI, 0.80-1.70], respectively; both from fixedeffect model with $I^{2}=0 \%$; Table 2 ), however, the number of studies ( $\mathrm{n}=3$ and 2 , respectively) that separately addressed these stroke types was small.

The corresponding absolute risk difference associated with depression based on the most recent stroke statistics for the United States ${ }^{45}$ was estimated to be 106 cases for total stroke, 53 cases for ischemic stroke, and 22 cases for fatal stroke per 100000 individuals per year. According to the most recent statistics, 9.0\% (21 million) of US adults meet the criteria for current depression, ${ }^{46}$ using the risk estimates from our meta-analysis, we estimated that $3.9 \%(n=273000)$ of stroke cases in the US were attributable to depression.

\section{Stratified and Sensitivity analyses}

Depression was associated with an increased risk of stroke in most subgroups (eFigure 2). The increased risk was more evident in several strata of study characteristics (Table 2): using clinical diagnosis to define depression, with a high study quality (more than the median score), having shorter follow-up ( $\leq 10$ years), featuring younger participants (mean age $<65$ years), having a relatively small study sample $(n<5000)$, with participants in Asians, and lack of statistical control for BMI or smoking status. Twenty-two reports (7334 cases) adjusted for smoking in the multivariate models, whereas 9 reports (1144 cases) did not. The pooled HR controlling for smoking $(1.28 ; 95 \% \mathrm{CI}, 1.21-1.36)$ was lower than pooled HR without smoking in the models (1.96; 95\% CI, 1.28-2.86). Likewise, the pooled HR of controlling for BMI $(1.28 ; 95 \%$ CI, 1.20-1.36; 15 reports, 6718 cases $)$ was lower than pooled HR without BMI in the models (1.76; 95\% CI, 1.33-2.32; 16 reports, 1760 cases). No between-group differences were observed for other variables (eTable 3). Nevertheless, moderate to high heterogeneities were observed in most of the subgroups.

\section{Analysis of Publication Bias}

Visual inspection of the funnel plot revealed asymmetry (eFigure 1A), and the Begg's test was significant $(\mathrm{z}=2.33 ; P=.02)$. A sensitivity analysis using the trim-and-fill method was performed with 6 imputed studies, which produced a symmetrical funnel plot (eFigure 1B). The pooled HR incorporating the 6 hypothetical studies was smaller than the original results, however, remained to be statistically significant (HR, 1.28; 95\% CI, 1.12-1.47; $P<.001)$. No significant publication bias was observed for fatal stroke $(P=.22)$, and a moderate bias for ischemic stroke ( $P=.04$; HR, 1.19 [95\% CI, 1.03-1.38] after trim and fill method).

\section{COMMENT}

The results of this meta-analysis demonstrate that the depression is prospectively associated with a significantly increased risk of developing stroke. Furthermore, the association persisted and remained statistically significant across several subgroups stratified by various 
study and participant characteristics. We also found a positive association of depression with fatal stroke and ischemic stroke.

Our results are consistent with a previous meta-analysis of 10 studies published before 2005 (HR, 1.43; 95\% CI, 1.17-1.75). ${ }^{9}$ Our current meta-analysis, with 5 times more cases, provides strong evidence that depression is associated with increased risks of total stoke, fatal stroke, and ischemic stroke. The result is also consistent with a large case-control study, the INTERSTROKE study ${ }^{47}$ where the investigators found that self-reported depression (for 2 or more weeks in the last year) was associated with a significantly increased risk of stroke (odds ratio, 1.35 [99\% CI, 1.10-1.66]) in 3000 cases and 3000 matched controls from 22 countries. Several studies that did not met the inclusion criteria for the meta-analysis also found a positive association between depression and stroke. For example, Simonsick et al. ${ }^{48}$ found that the stroke incident rates were 2.3 to 2.7 times higher in most subgroups with high depressive symptoms compared with their non-depressed counterparts in a population of older adults with hypertension $(n=3461)$; Nilsson and Kessing ${ }^{49}$ found that patients with depression severe enough $(n=11741)$ to be hospitalized was associated with an increased future risk of stroke (HR, 1.22; 95\% CI, 1.06-1.41) compared with patients with osteoarthritis $(\mathrm{n}=81380)$ in Denmark. Using a continuous variable of 20-item CES-D score, Ostir et al. ${ }^{50}$ found that depressive symptoms were associated with an increased stroke risk (HR, 1.01 per score increase; 95\% CI, 1.00-1.02) in 2682 Mexican Americans aged 65 years and older.

Depression may contribute to stroke through a variety of mechanisms. First, depression has known neuroendocrine (e.g., sympathetic nervous system activation, dysregulation of the hypothalamic-pituitary-adrenocortical axis, platelet aggregation dysfunction) ${ }^{6}$ and immunological/inflammation effects, ${ }^{51}$ which could influence stroke risk. A recent metaanalysis suggests that depression is positively associated with C-reactive protein (CRP), interleukin (IL)-1, and IL-6 in clinical and community samples, ${ }^{52}$ and these inflammatory factors have been associated with an increased risk of stroke. ${ }^{53}$ Second, depression is associated with poor health behaviors (i.e., smoking, physical inactivity, poor diet, lack of medication compliance $)^{54}$ and obesity, ${ }^{55}$ which might increase the risk of stroke. Adjusting for smoking or BMI somewhat attenuated the association between depression and stroke, suggesting that smoking and obesity may confound or mediate the association between depression and stroke. The magnitude of the depression-stroke association observed in this study is similar to the associations between smoking and stroke (HR, 1.51; 95\% CI, 1.451.58; from a meta-analysis) ${ }^{56}$ between obesity and stroke (HR, 1.26 ; $95 \%$ CI, $1.07-1.48$; from a meta-analysis) ${ }^{57}$ Third, depression is correlated with other major comorbidities, such as diabetes ${ }^{4}$ and hypertension, ${ }^{5}$ both of which are major risk factors for stroke. Finally, antidepressant medication use may contribute to the observed association. We found a positive association between the medication use and stroke risk, however, the results should be interpreted cautiously because medication use can be marker of depression severity, and many studies lacked information on dose and duration of medication use.

Several limitations of this meta-analysis should be considered. First, we found a significant heterogeneity across studies, which may result from differences in study designs, sample sizes, depression and stroke measures, analysis strategies, and participants' characteristics. Although moderate to high heterogeneities still remained in many subgroups, the pooled HRs showed consistent positive associations in most subgroups. Second, the funnel plot indicated a possible publication bias; however, the trim-and-fill sensitivity analysis did not materially change the results (although the pooled HR was modestly attenuated).

Nevertheless, the possibility of publication bias could not be fully excluded by this method. Moreover, the meta-analysis was limited to English publications, and the possibility of unpublished reports was not yet identified. Data extraction and analyses were not blinded to 
the authors, journals or institutions of the publications; however, the literature screening and data extraction were conducted independently by two investigators, and thus, the selection bias was unlikely. Furthermore, most studies did not have information on depression treatment and antidepressant medication use. The role of depression treatment in modulating subsequent risk of stroke needs to be studied further. Finally, further studies are needed to determine whether depression is associated with hemorrhagic stroke.

In conclusion, this meta-analysis provides strong evidence that depression is a significant risk factor for stroke. Given the high prevalence and incidence of depression and stroke in the general population, the observed association between depression and stroke has significant clinical and public health importance. More studies are needed to explore the underlying mechanisms and elucidate the causal pathways that link depression and stroke.

\section{Supplementary Material}

Refer to Web version on PubMed Central for supplementary material.

\section{Acknowledgments}

We are grateful for Dr. Lonneke Wouts, MD, from Departments of Psychiatry, Radboud University Nijmegen Medical Centre, the Netherlands, for providing unpublished data, and Dr. Til Stürmer, MD, MPH, from School of Public Health, University of North Carolina at Chapel Hill, United States, for clarifying inquiry of his papers. None of them were compensated.

Funding/Support: The project was supported by NIH grants HL034594 and HL088521. Dr. Sun was supported by a career development award K99HL098459 from the National Heart, Lung, and Blood Institute. Dr. Okereke was supported by the NIH (career development award K08AG029813 from National Institute of Aging, and R01MH091448 from the National Institute of Mental Health).

Role of the Sponsor: None of the funding sponsors was involved in the design and conduct of the study; collection, management, analysis, and interpretation of the data; and preparation, review, or approval of the manuscript.

\section{References}

1. Goldstein LB, Bushnell CD, Adams RJ, et al. Guidelines for the Primary Prevention of Stroke: A Guideline for Healthcare Professionals From the American Heart Association/American Stroke Association. Stroke. 2011; 42(2):517-584. [PubMed: 21127304]

2. World Health Organization. The World Health Report 2001 - Mental Health: New Understanding, New Hope. Geneve, Switzerland: 2001.

3. Kessler RC, Berglund P, Demler O, et al. The epidemiology of major depressive disorder: results from the National Comorbidity Survey Replication (NCS-R). JAMA. 2003; 289(23):3095-3105. [PubMed: 12813115]

4. Pan A, Lucas M, Sun Q, et al. Bidirectional association between depression and type 2 diabetes mellitus in women. Arch Intern Med. 2010; 170(21):1884-1891. [PubMed: 21098346]

5. Patten SB, Williams JVA, Lavorato DH, Campbell NRC, Eliasziw M, Campbell TS. Major Depression as a Risk Factor for High Blood Pressure: Epidemiologic Evidence From a National Longitudinal Study. Psychosom Med. 2009; 71(3):273-279. [PubMed: 19196807]

6. Musselman DL, Evans DL, Nemeroff CB. The relationship of depression to cardiovascular disease: epidemiology, biology, and treatment. Arch Gen Psychiatry. 1998; 55(7):580-592. [PubMed: 9672048]

7. Ramasubbu R, Patten SB. Effect of depression on stroke morbidity and mortality. Can J Psychiatry. 2003; 48(4):250-257. [PubMed: 12776392]

8. Thomas AJ, Kalaria RN, O'Brien JT. Depression and vascular disease: what is the relationship? J Affect Disord. 2004; 79(1-3):81-95. [PubMed: 15023483] 
9. Van der Kooy K, van Hout H, Marwijk H, Marten H, Stehouwer C, Beekman A. Depression and the risk for cardiovascular diseases: systematic review and meta analysis. Int J Geriatr Psychiatry. 2007; 22(7):613-626. [PubMed: 17236251]

10. Higgins JP, Thompson SG. Quantifying heterogeneity in a meta-analysis. Stat Med. 2002; 21(11): 1539-1558. [PubMed: 12111919]

11. Higgins JP, Thompson SG, Deeks JJ, Altman DG. Measuring inconsistency in meta-analyses. BMJ. 2003; 327(7414):557-560. [PubMed: 12958120]

12. DerSimonian R, Laird N. Meta-analysis in clinical trials. Control Clin Trials. 1986; 7(3):177-188. [PubMed: 3802833]

13. Begg CB, Mazumdar M. Operating characteristics of a rank correlation test for publication bias. Biometrics. 1994; 50(4):1088-1101. [PubMed: 7786990]

14. Egger M, Davey Smith G, Schneider M, Minder C. Bias in meta-analysis detected by a simple, graphical test. BMJ. 1997; 315(7109):629-634. [PubMed: 9310563]

15. Duval S, Tweedie R. Trim and fill: A simple funnel-plot-based method of testing and adjusting for publication bias in meta-analysis. Biometrics. 2000; 56(2):455-463. [PubMed: 10877304]

16. Simons LA, McCallum J, Friedlander Y, Simons J. Risk Factors for Ischemic Stroke: Dubbo Study of the Elderly. Stroke. 1998; 29(7):1341-1346. [PubMed: 9660384]

17. Whooley MA, Browner WS. Association between depressive symptoms and mortality in older women. Study of Osteoporotic Fractures Research Group. Arch Intern Med. 1998; 158(19):2129_ 2135. [PubMed: 9801180]

18. Pan A, Okereke O, Sun Q, et al. Depression and incident stroke in women. Stroke. 2011 in press.

19. Vogt T, Pope C, Mullooly J, Hollis J. Mental health status as a predictor of morbidity and mortality: a 15-year follow-up of members of a health maintenance organization. Am J Public Health. 1994; 84(2):227-231. [PubMed: 8296945]

20. Wassertheil-Smoller S, Applegate WB, Berge K, et al. Change in depression as a precursor of cardiovascular events. SHEP Cooperative Research Group (Systoloc Hypertension in the elderly). Arch Intern Med. 1996; 156(5):553-561. [PubMed: 8604962]

21. Everson SA, Roberts RE, Goldberg DE, Kaplan GA. Depressive symptoms and increased risk of stroke mortality over a 29-year period. Arch Intern Med. 1998; 158(10):1133-1138. [PubMed: 9605786]

22. Jonas BS, Mussolino ME. Symptoms of depression as a prospective risk factor for stroke. Psychosom Med. 2000; 62(4):463-471. [PubMed: 10949089]

23. Larson SL, Owens PL, Ford D, Eaton W. Depressive disorder, dysthymia, and risk of stroke: thirteen-year follow-up from the Baltimore Epidemiologic Catchment Area Study. Stroke. 2001; 32(9):1979-1983. [PubMed: 11546884]

24. Ohira T, Iso H, Satoh S, et al. Prospective study of depressive symptoms and risk of stroke among Japanese. Stroke. 2001; 32(4):903-908. [PubMed: 11283390]

25. Ostir GV, Markides KS, Peek MK, Goodwin JS. The association between emotional well-being and the incidence of stroke in older adults. Psychosom Med. 2001; 63(2):210-215. [PubMed: 11292267]

26. May M, McCarron P, Stansfeld S, et al. Does psychological distress predict the risk of ischemic stroke and transient ischemic attack? The Caerphilly Study. Stroke. 2002; 33(1):7-12. [PubMed: 11779881]

27. Yasuda N, Mino Y, Koda S, Ohara H. The differential influence of distinct clusters of psychiatric symptoms, as assessed by the general health questionnaire, on cause of death in older persons living in a rural community of Japan. J Am Geriatr Soc. 2002; 50(2):313-320. [PubMed: 12028214]

28. Wassertheil-Smoller S, Shumaker S, Ockene J, et al. Depression and cardiovascular sequelae in postmenopausal women. The Women's Health Initiative (WHI). Arch Intern Med. 2004; 164(3): 289-298. [PubMed: 14769624]

29. Gump BB, Matthews KA, Eberly LE, Chang YF. Depressive symptoms and mortality in men: results from the Multiple Risk Factor Intervention Trial. Stroke. 2005; 36(1):98-102. [PubMed: 15569872] 
30. Avendano M, Kawachi I, Lenthe FV, et al. Socioeconomic status and stroke incidence in the US elderly: The role of risk factors in the EPESE study. Stroke. 2006; 37(6):1368-1373. [PubMed: 16690902]

31. Stürmer T, Hasselbach P, Amelang M. Personality, lifestyle, and risk of cardiovascular disease and cancer: follow-up of population based cohort. BMJ. 2006; 332(7554):1359. [PubMed: 16687457]

32. Arbelaez JJ, Ariyo AA, Crum RM, Fried LP, Ford DE. Depressive symptoms, inflammation, and ischemic stroke in older adults: a prospective analysis in the cardiovascular health study. $\mathrm{J} \mathrm{Am}$ Geriatr Soc. 2007; 55(11):1825-1830. [PubMed: 17916124]

33. Kawamura T, Shioiri T, Takahashi K, Ozdemir V, Someya T. Survival rate and causes of mortality in the elderly with depression: a 15-year prospective study of a Japanese community sample, the Matsunoyama-Niigata suicide prevention project. J Investig Med. 2007; 55(3):106-114.

34. Salaycik KJ, Kelly-Hayes M, Beiser A, et al. Depressive symptoms and risk of stroke: the Framingham Study. Stroke. 2007; 38(1):16-21. [PubMed: 17138952]

35. Bos MJ, Linden T, Koudstaal PJ, et al. Depressive symptoms and risk of stroke: the Rotterdam Study. J Neurol Neurosurg Psychiatry. 2008; 79(9):997-1001. [PubMed: 18208858]

36. Lee HC, Lin HC, Tsai SY. Severely depressed young patients have over five times increased risk for stroke: a 5-year follow-up study. Biol Psychiatry. 2008; 64(10):912-915. [PubMed: 18718571]

37. Liebetrau M, Steen B, Skoog I. Depression as a risk factor for the incidence of first-ever stroke in 85-year-olds. Stroke. 2008; 39(7):1960-1965. [PubMed: 18451342]

38. Surtees PG, Wainwright NW, Luben RN, Wareham NJ, Bingham SA, Khaw KT. Psychological distress, major depressive disorder, and risk of stroke. Neurology. 2008; 70(10):788-794. [PubMed: 18316690]

39. Whooley MA, de Jonge P, Vittinghoff E, et al. Depressive symptoms, health behaviors, and risk of cardiovascular events in patients with coronary heart disease. JAMA. 2008; 300(20):2379-2388. [PubMed: 19033588]

40. Wouts L, Oude Voshaar RC, Bremmer MA, Buitelaar JK, Penninx BW, Beekman AT. Cardiac disease, depressive symptoms, and incident stroke in an elderly population. Arch Gen Psychiatry. 2008; 65(5):596-602. [PubMed: 18458211]

41. Glymour MM, Maselko J, Gilman SE, Patton KK, Avendano M. Depressive symptoms predict incident stroke independently of memory impairments. Neurology. 2010; 75(23):2063-2070. [PubMed: 21135381]

42. Nabi H, Kivimaki M, Suominen S, Koskenvuo M, Singh-Manoux A, Vahtera J. Does depression predict coronary heart disease and cerebrovascular disease equally well? The Health and Social Support Prospective Cohort Study. Int J Epidemiol. 2010; 39(4):1016-24. [PubMed: 20360321]

43. Peters R, Pinto E, Beckett N, et al. Association of depression with subsequent mortality, cardiovascular morbidity and incident dementia in people aged 80 and over and suffering from hypertension. Data from the Hypertension in the Very Elderly Trial (HYVET). Age Ageing. 2010; 39(4):439-445. [PubMed: 20497949]

44. Smoller JW, Allison M, Cochrane BB, et al. Antidepressant use and risk of incident cardiovascular morbidity and mortality among postmenopausal women in the Women's Health Initiative study. Arch Intern Med. 2009; 169(22):2128-2139. [PubMed: 20008698]

45. Roger VL, Go AS, Lloyd-Jones DM, et al. for the American Heart Association Statistics Committee and Stroke Statistics Subcommittee. Heart disease and stroke statistics-2011 update: a report from the American Heart Association. 2011; 123(4):e18-e209.

46. Centers for Disease Control and Prevention (CDC). Current depression among adults-United States, 2006 and 2008. MMWR Morb Mortal Wkly Rep. 2010; 59(38):1229-1235. [PubMed: 20881934]

47. O'Donnell MJ, Xavier D, Liu L, et al. Risk factors for ischaemic and intracerebral haemorrhagic stroke in 22 countries (the INTERSTROKE study): a case-control study. Lancet. 2010; 376(9735): 112-123. [PubMed: 20561675]

48. Simonsick E, Wallace R, Blazer D, Berkman L. Depressive symptomatology and hypertensionassociated morbidity and mortality in older adults. Psychosom Med. 1995; 57(5):427-435. [PubMed: 8552732] 
49. Nilsson FM, Kessing LV. Increased risk of developing stroke for patients with major affective disorder--a registry study. Eur Arch Psychiatry Clin Neurosci. 2004; 254(6):387-391. [PubMed: 15538602]

50. Ostir GV, Raji MA, Ottenbacher KJ, Markides KS, Goodwin JS. Cognitive function and incidence of stroke in older Mexican Americans. J Gerontol A Biol Sci Med Sci. 2003; 58(6):531-5. [PubMed: 12807924]

51. Shimbo D, Chaplin W, Crossman D, Haas D, Davidson KW. Role of depression and inflammation in incident coronary heart disease events. Am J Cardiol. 2005; 96(7):1016-1021. [PubMed: 16188535]

52. Howren MB, Lamkin DM, Suls J. Associations of depression with C-reactive protein, IL-1, and IL-6: a meta-analysis. Psychosom Med. 2009; 71(2):171-186. [PubMed: 19188531]

53. Kaptoge S, Di Angelantonio E, Lowe G, et al. Emerging Risk Factors Collaboration. C-reactive protein concentration and risk of coronary heart disease, stroke, and mortality: an individual participant meta-analysis. Lancet. 2010; 375(9709):132-140. [PubMed: 20031199]

54. Strine TW, Mokdad AH, Dube SR, et al. The association of depression and anxiety with obesity and unhealthy behaviors among community-dwelling US adults. Gen Hosp Psychiatry. 2008; 30(2):127-137. [PubMed: 18291294]

55. Luppino FS, de Wit LM, Bouvy PF, et al. Overweight, obesity, and depression: a systematic review and meta-analysis of longitudinal studies. Arch Gen Psychiatry. 2010; 67(3):220-229. [PubMed: 20194822]

56. Shinton R, Beevers G. Meta-analysis of relation between cigarette smoking and stroke. BMJ. 1989; 298(6676):789-794. [PubMed: 2496858]

57. Strazzullo P, D'Elia L, Cairella G, Garbagnati F, Cappuccio FP, Scalfi L. Excess body weight and incidence of stroke: meta-analysis of prospective studies with 2 million participants. Stroke. 2010; 41(5):e418-426. [PubMed: 20299666] 


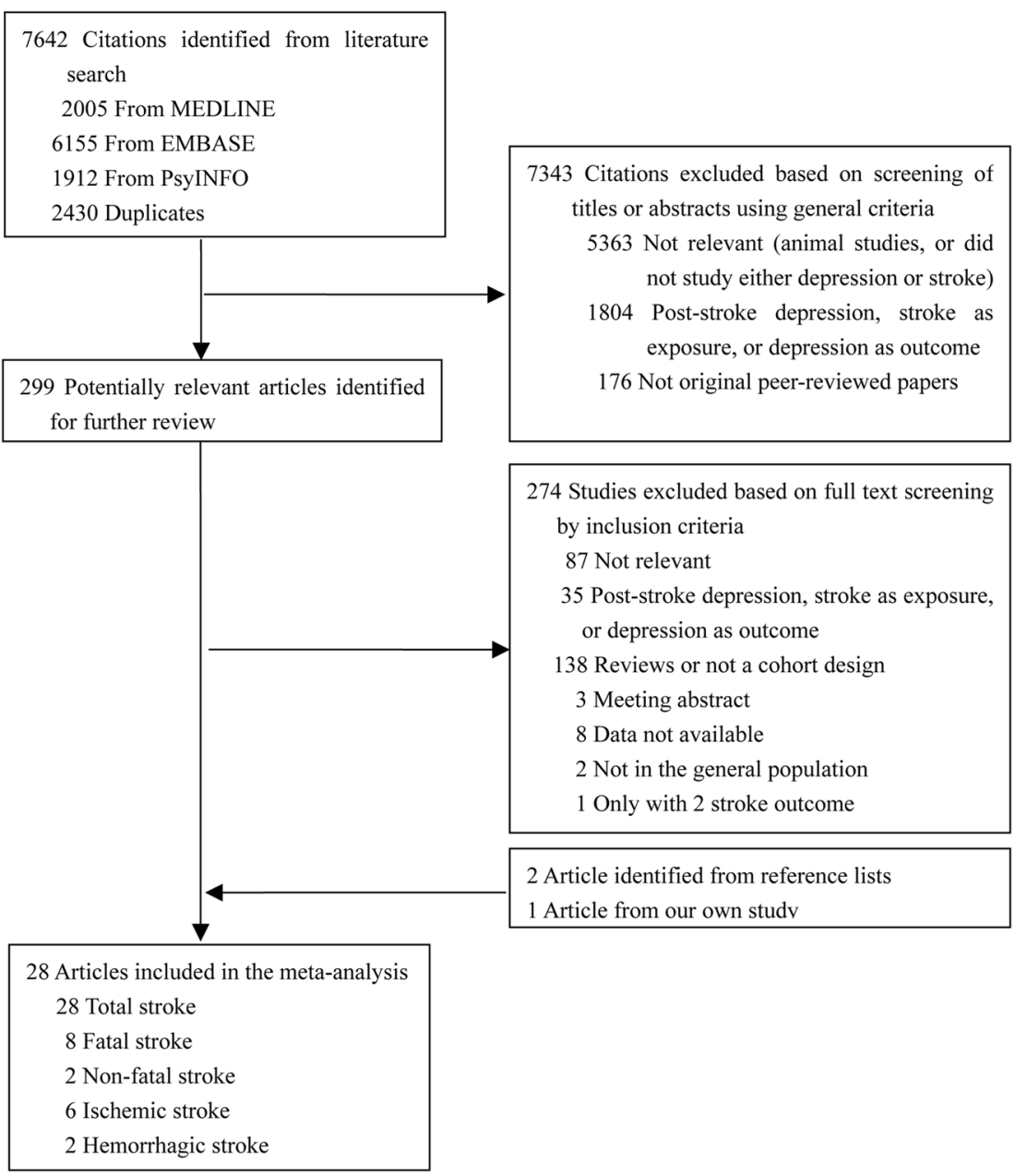

Figure 1.

Flowchart of the Meta-analysis 


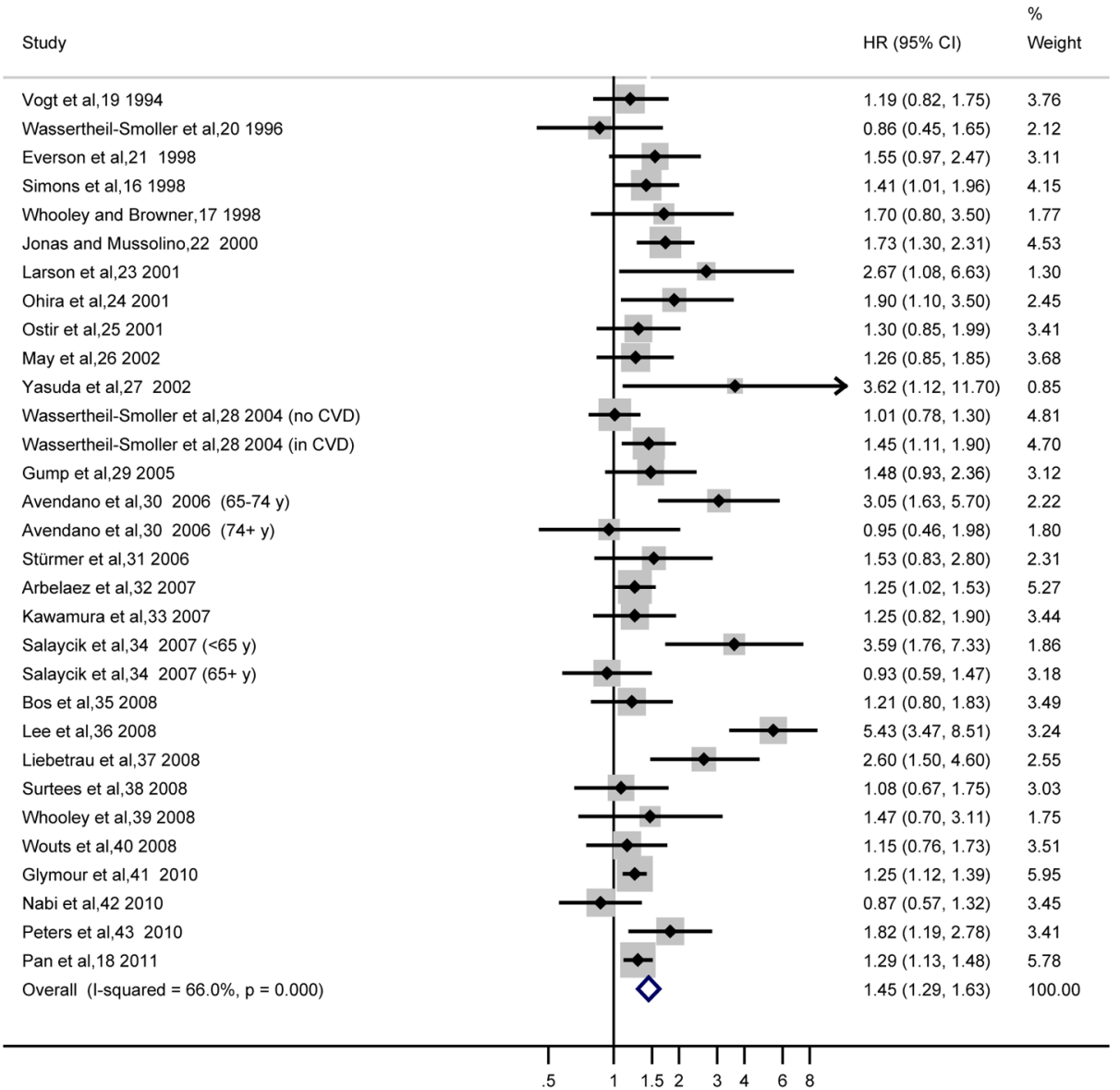

Figure 2.

Adjusted Hazard Ratios of Total Stroke for Depressed Participants Compared with Nondepressed Individuals

The summary estimates were obtained using a random-effects model. The data markers indicate the adjusted hazard ratios (HRs) in depressed participants compared with nondepressed individuals. The size of the data markers indicates the weight of the study, which is the inverse variance of the effect estimate. The diamond data markers indicate the pooled HRs. CI indicates confidence interval. 


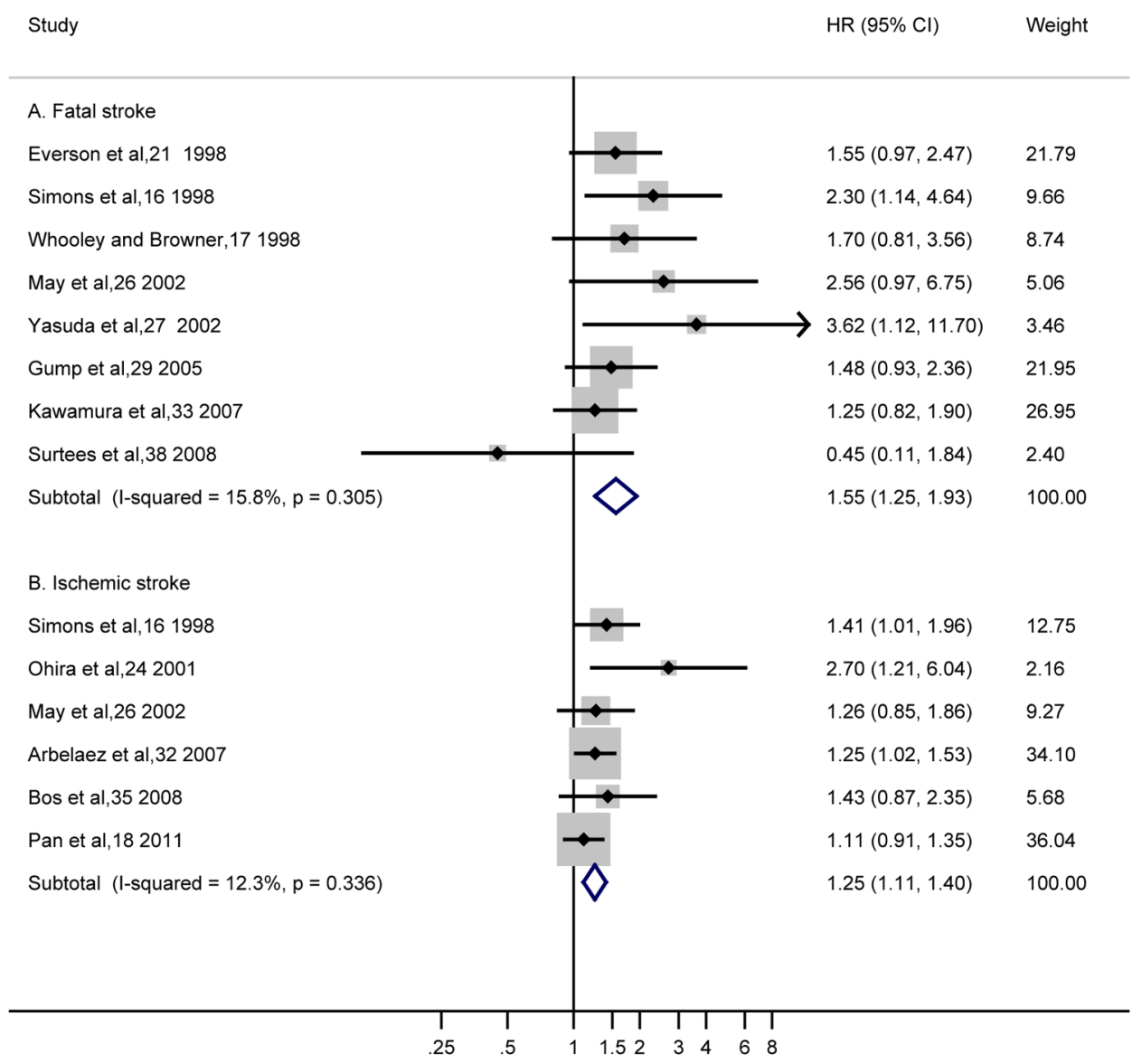

Figure 3.

Adjusted Hazard Ratios of (A) Fatal Stroke and (B) Ischemic Stroke for Depressed Participants Compared with Non-depressed Individuals

The summary estimates were obtained using a fixed-effect model. The data markers indicate the adjusted hazard ratios (HRs) in depressed participants compared with non-depressed individuals. The size of the data markers indicates the weight of the study, which is the inverse variance of the effect estimate. The diamond data markers indicate the pooled HRs. CI indicates confidence interval. 


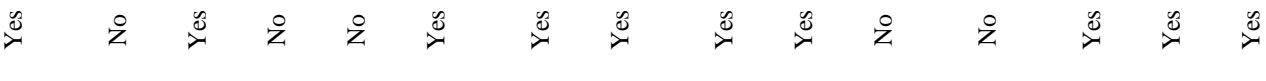

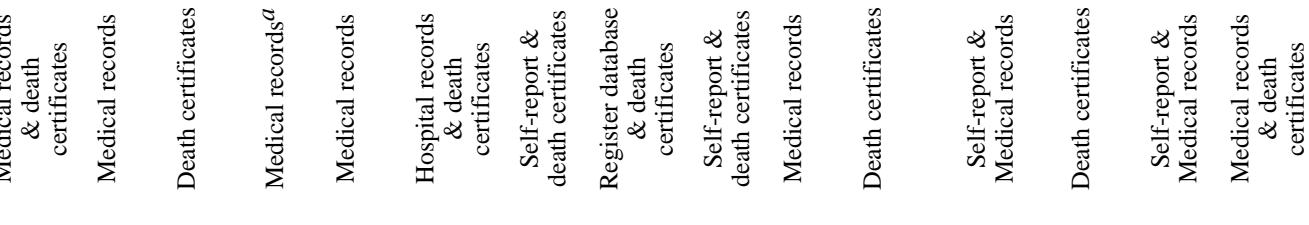

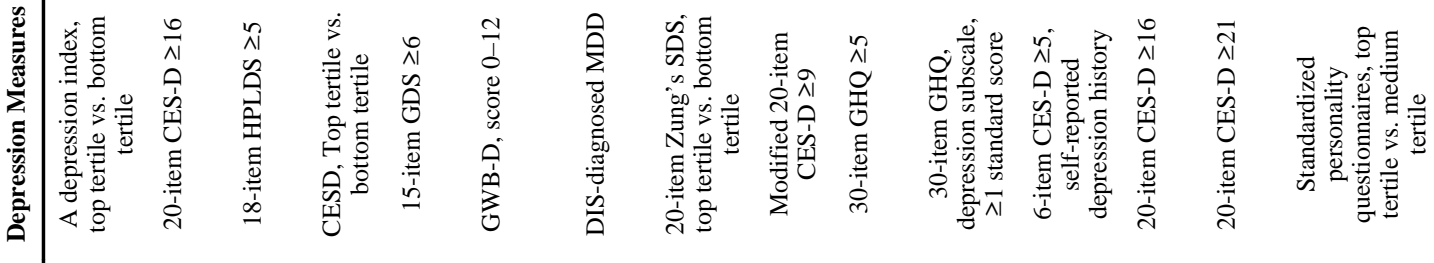

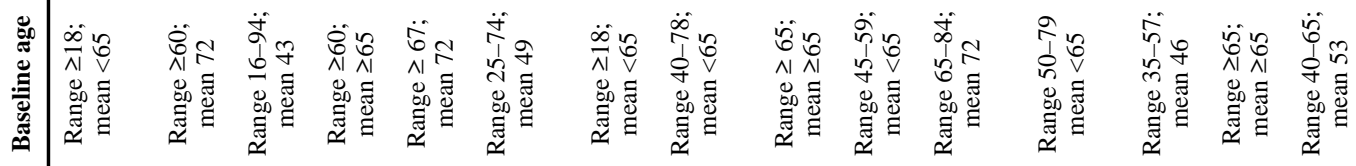
竞

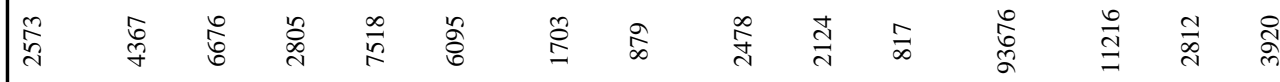




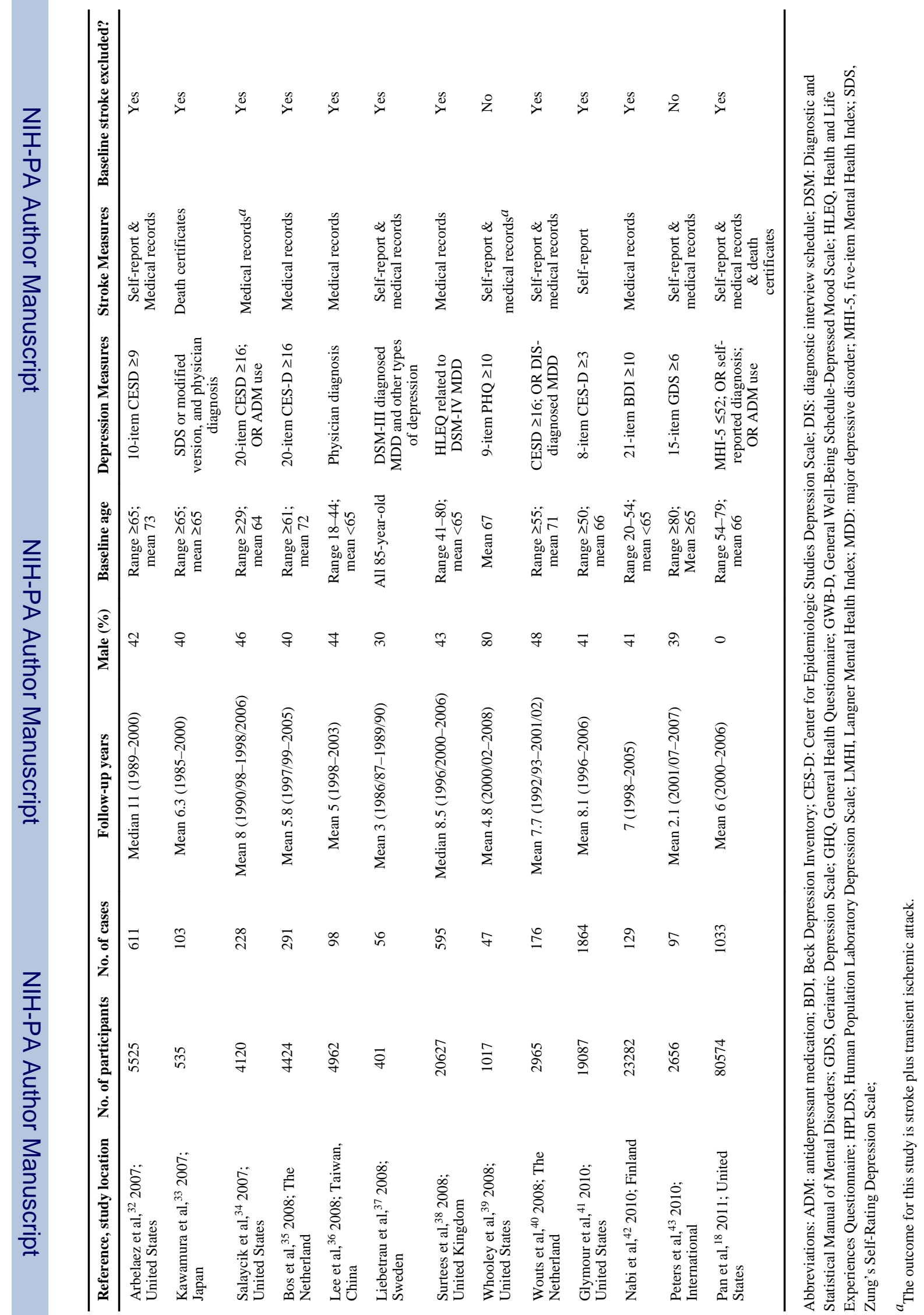

JAMA. Author manuscript; available in PMC 2012 September 21. 


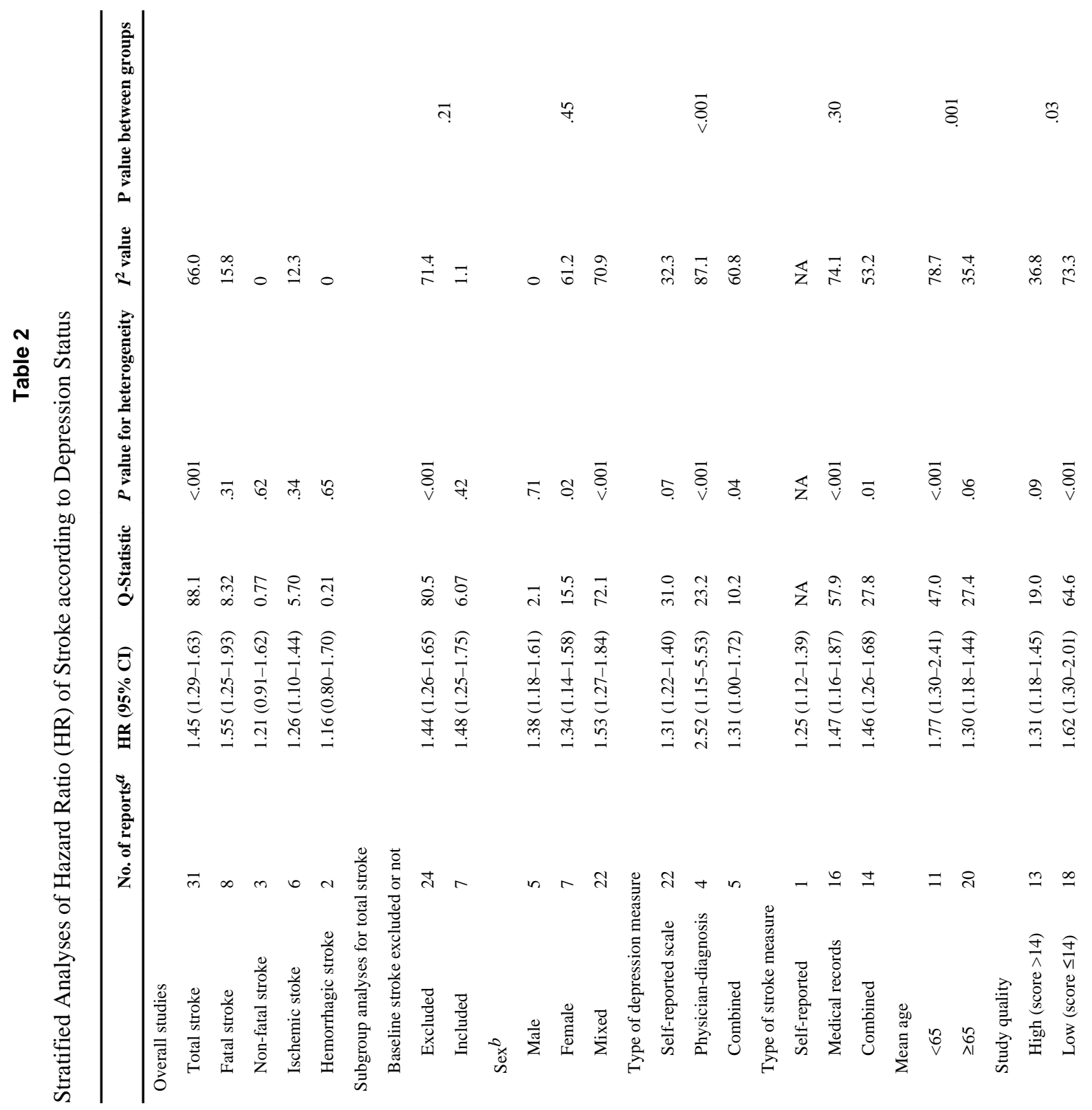




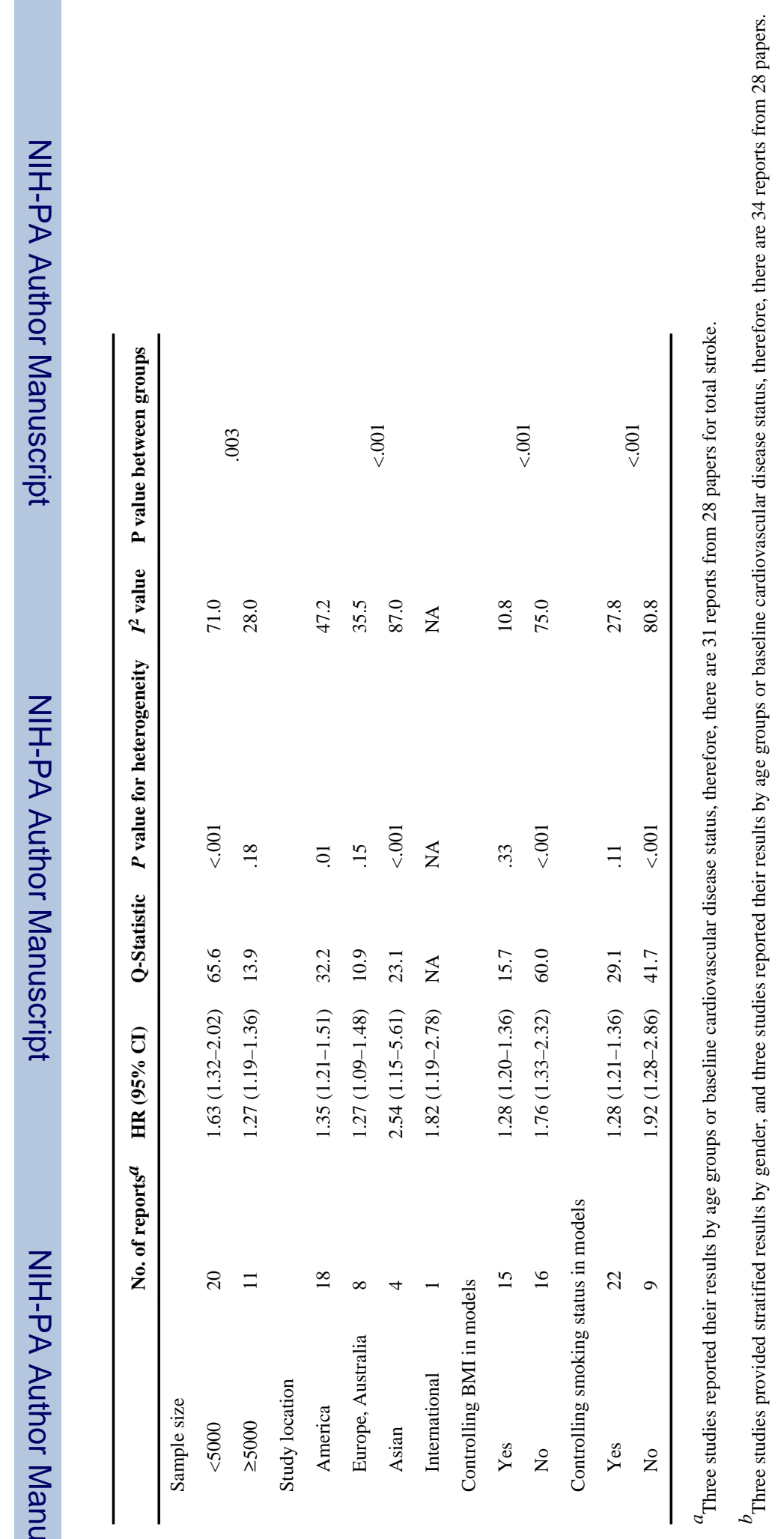

